\title{
ONLINE INTEGRATION OF OPTIMAL
}

CLEANING SCHEDULING AND CONTROL

OF HEAT EXCHANGER NETWORKS

\section{UNDER FOULING - SUPPLEMENTARY}

\section{MATERIAL.}

Federico Lozano Santamaria* \& Sandro Macchietto.

Department of Chemical Engineering, Imperial College London, South Kensington

Campus, London SW7 2AZ, UK

Keywords: crude oil fouling, scheduling and control integration, NMPC, online optimization.

* Author for correspondence (f13015@ic.ac.uk) 


\section{Nomenclature}

Subscripts
cl
flow
$f$
$i$
$o$
$o p$
$s$
$t$

\begin{tabular}{lll}
\multicolumn{1}{c}{ Symbol } & \multicolumn{1}{c}{ Units } & \\
$\alpha$ & {$\left[\mathrm{m}^{2} \mathrm{~K} / \mathrm{W}\right.$. day $]$} & Deposition parameter \\
$\gamma$ & {$\left[\mathrm{kg} / \mathrm{m}^{2} \mathrm{~W}\right.$. day.Pa $]$} & Removal parameter \\
$\delta$ & {$[\mathrm{mm}]$} & Deposit thickness \\
$\epsilon$ & {$[-]$} & Regularization parameter for relaxation \\
$\Delta P$ & {$[\mathrm{bar}]$} & Pressure drop \\
$\lambda$ & {$[\mathrm{W} / \mathrm{m} \cdot \mathrm{K}]$} & Thermal conductivity \\
$\rho$ & {$\left[\mathrm{kg} / \mathrm{m}^{3}\right]$} & Density \\
$\tau$ & {$[\mathrm{days}]$} & Period duration \\
$\tau_{w}$ & {$[\mathrm{~Pa}]$} & Shear stress \\
$A$ & {$\left[\mathrm{~m}{ }^{2}\right]$} & Transfer area \\
$C_{P}$ & {$[\mathrm{~kJ} / \mathrm{kg} \cdot \mathrm{K}]$} & Specific heat capacity \\
$E$ & {$[\mathrm{~J} / \mathrm{mol} \cdot \mathrm{K}]$} & Activation energy \\
$f$ & {$[-]$} & Friction factor \\
$G$ & {$\left[\mathrm{~kg} / \mathrm{m}^{2} \mathrm{~s}\right]$} & Mass flux rate \\
$h$ & {$\left[\mathrm{~W} / \mathrm{m}^{2} \cdot \mathrm{K}\right]$} & Convective heat transfer coefficient \\
$k^{0}$ & {$[1 / \mathrm{s}]$} & Pre-exponential factor \\
$L$ & {$[\mathrm{~m}]$} & Heat exchanger tube length \\
$m$ & {$[\mathrm{~kg} / \mathrm{s}]$} & Mass flow rate \\
$N_{P}$ & {$[-]$} & Number of passes \\
$N T U$ & {$[-]$} & Number of transfer units \\
$P$ & {$[-]$} & Efficiency of heat transfer with respect to the tube side \\
$P r$ & {$[-]$} & Prandtl number \\
$Q$ & {$[\mathrm{~W}]$} & Heat transfer rate \\
$R$ & {$[\mathrm{~mm}]$} & Radius \\
$R_{C}$ & {$[-]$} & Heat capacity ratio \\
$R_{f}$ & {$\left[\mathrm{~m}{ }^{2} \mathrm{~K} / \mathrm{W}\right]$} & Fouling thermal resistance \\
$R e$ & {$[-]$} & Reynolds number \\
& &
\end{tabular}

Cleaning mode

Condition at the flow boundary (fluid - deposit)

Film

Inner side of the tube

Outer side of the tube

Operating mode

Shell side

Tube side

\section{Description}




$\begin{array}{lll}s_{a}, S_{b} & {[-]} & \text { Slack variables } \\ t & {[\mathrm{day}]} & \text { Time } \\ T & {[\mathrm{~K}]} & \text { Temperature } \\ T_{t} & {[\text { days }]} & \text { Starting time of period } t \\ U & {\left[\mathrm{~W} / \mathrm{m}^{2} . \mathrm{K}\right]} & \text { Overall heat transfer coefficient } \\ y & {[-]} & \text { Binary variable to switch operation modes }\{0,1\} \\ Y & {[-]} & \text { Logic variable }\{\text { True, False }\}\end{array}$




\section{Models of heat exchangers and fouling}

This section presents in detail the equations that define each of the components of the heat exchange network under fouling model. The equations are presented for completeness, but the model was originally presented in [1] - Reprinted (adapted) with permission from $F$. Lozano Santamaria and S. Macchietto, "Integration of Optimal Cleaning Scheduling and Control of Heat Exchanger Networks Undergoing Fouling: Model and Formulation,"' Ind. Eng. Chem. Res., vol. 57, no. 38, pp. 12842-12860, Sep. 2018. Copyright (2018) American Chemical Society - while the solution strategy for the optimal integration of cleaning scheduling and flow control was presented in [2].

In this model it is assumed that: 1) axial effects are neglected and averaged over the length, 2) the physical properties are function of temperature for each stream, hence they change with time, 3) heat transfers rates and coefficients averaged between inlet and outlet conditions, 4) operation is pseudo steady state, and 5) streams are single phase.

The following sets are defined for a compact notation of the model equations:

- $H E X=\left\{1,2, \ldots, n_{H E X}\right\}$. Set of heat exchangers in the network.

- $S p=\left\{1,2, \ldots, n_{S p}\right\}$. Set of splitters in the network.

- $M x=\left\{1,2, \ldots, n_{M x}\right\}$. Set of mixers in the network.

- $\quad S o=\left\{1,2, \ldots, n_{S o}\right\}$. Set of source nodes.

- $S i=\left\{1,2, \ldots, n_{S i}\right\}$. Set of sink nodes.

- $\quad$ Nodes $=H E X \cup S p \cup M x \cup S o \cup S i$. Set of all the nodes in the network. 
- Stream types $=\left\{1,2, \ldots, n_{F}\right\}$. Set of stream types in the network, where each type is a different fluid.

- $\operatorname{Arcs}=\{(i, j, k) \mid \exists(i, j, k) \in$ Nodes $\times$ Nodes $\times$ Stream types $\}$. Set of arcs that define the connection between nodes for a given stream type.

- Periods $=\left\{1,2, \ldots, n_{P}\right\}$. Set of periods

- Points $=\left\{1,2, \ldots, n_{t}\right\}$. Set of internal points in the periods

The set Steam types refers to the nature of the streams (e.g. crude oil, residue), and the set Arcs includes every connection that exist in the network between nodes. The sets Periods and Points are used to represent the time discretization. In a discrete time approach the number of points per periods is one, and the length of each period is constant, while in a continuous time approach the number of points is the number of discretization points using orthogonal collocation in finite elements and the length of the periods is variable. The following model is formulated assuming a continuous time discretization, although the only difference with respect to a discrete time formulation is the cardinality of the set Points, and nature of the length of the periods.

Mass balances. Mass is conserved in each node and there is no accumulation.

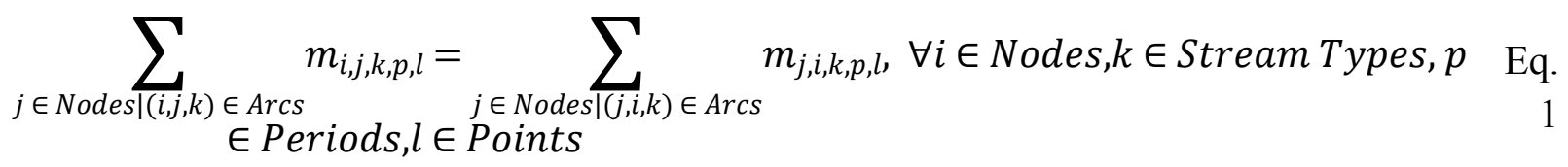

Energy balances. Energy is conserved in each node and there is no accumulation. The energy balance is simplified in terms of the enthalpy of the streams.

$$
\sum_{j \in \operatorname{Nodes}(i, j, k) \in \operatorname{Arcs}}^{\in} m_{i, j, k, p, l} C p_{k} T_{i, j, k, p, l}=\sum_{\substack{j \in \text { Nodes } \mid(j, i, k) \in \operatorname{Arcs} \\ \in\{x}} m_{j, i, k, p, l} C \operatorname{Sp}_{k} T_{j, i, k, p, l}, \forall i
$$


Inlet temperature at source nodes (included in 'Energy balances'). The temperature at source nodes is assumed fixed and cannot be controlled. This formulation assumes that there is only one arc between each source node and one other node in the network.

$\sum_{j \in \operatorname{Nodes} \mid(i, j, k) \in \operatorname{Arcs}} T_{i, j, k, p, l}=T_{i}^{0}, \forall i \in$ So,k $\in$ Stream Types, $p \in$ Periods,$l \in$ Points $\begin{array}{r}\text { Eq. } \\ 3\end{array}$

Outlet temperature of the splitters (included in 'Energy balances'). The splitters operate adiabatically, and all the outlet streams temperatures are equal to the inlet temperature.

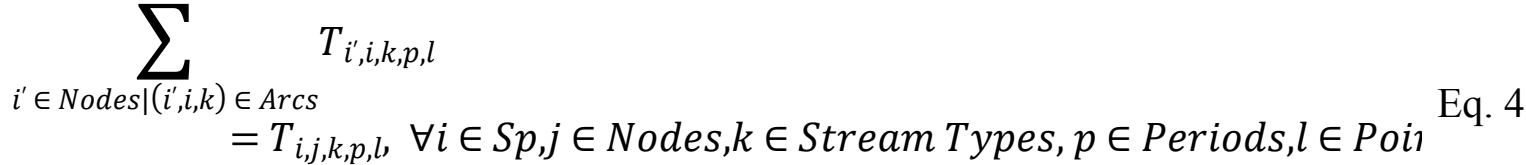

$$
\begin{aligned}
& i, j, k) \in \operatorname{Arcs}
\end{aligned}
$$

Assignment of the state of the exchanger among the types of cleanings (included in 'Cleaning sequence constraints'). If a cleaning task among all those possible is chosen for an exchanger, the state of the exchanger is "being cleaned", otherwise if no cleaning is chosen the state of the exchanger is "operating".

$$
\underline{\vee}\left(W_{p, i, c} \forall c \in \text { Cleaning types }\right) \Rightarrow Y_{p, i}, \forall i \in H E X, p \in \text { Periods } \backslash\left\{n_{p}\right\} \quad \text { Eq. } 5
$$

Assignment of the state of the network (included in 'Cleaning sequence constraints'). If one or more exchanges are being cleaned in a period, using any cleaning alternative, the state of the whole network is defined as "being cleaned" otherwise is defined as "operating".

$$
\underline{\mathrm{\vee}}\left(Y_{p, i} \forall i \in H E X\right) \Longrightarrow Z_{p}, \forall p \in \operatorname{Periods} \backslash\left\{n_{p}\right\} \quad \text { Eq. } 6
$$

No consecutive cleanings of the same unit (included in 'Cleaning sequence constraints'). This constraint is not required in the mathematical model, but it helps to prune solutions that are known a priori to be non-optimal. This apply to any type of cleanings. 
$Y_{p, i} \Rightarrow \neg Y_{p+1, i}, \forall i \in H E X, p \in$ Periods $\backslash\left\{n_{p}\right\}$

Eq. 7

Non-decreasing starting time of the events (included in 'Continuous time representation')

$$
\begin{gathered}
t_{p}^{S}=0, \forall p \in\{1\} \\
t_{p}^{S} \geq t_{p-1}^{S}, \forall p \in \text { Periods } \backslash\{1\}
\end{gathered}
$$

Duration of each period (included in 'Continuous time representation')

$$
\begin{gathered}
\tau_{p}=t_{p+1}^{S}-t_{p}^{S}, \forall p \in \text { Periods } \backslash\left\{n_{p}\right\} \\
\tau_{p}=t^{f}-t_{p}^{S}, \forall p \in\left\{n_{p}\right\}
\end{gathered}
$$

Disjunction for the duration of the period, depending on the mode of operation (included in 'Disjunctions for operating mode'). There are two modes of operation for the network: "being cleaned" (when at least one exchanger is being cleaned by one cleaning type) and "operating", depending on which the duration of the period is set as the corresponding cleaning time (here, fixed a priori) or is a free decision variable.

$$
\left[\tau_{p}=\max _{i \in H E X, c \in \text { Cleaning types }}^{Z_{p}}\left\{\left[\begin{array}{c}
W_{p, i, c} \\
t_{c l, i, c}
\end{array}\right] \vee\left[\begin{array}{c}
\neg W_{p, i, c} \\
0
\end{array}\right]\right\}\right] \vee\left[\begin{array}{c}
\neg Z_{p} \\
\tau_{o p}^{\min } \leq \tau_{p} \leq t_{o p}^{\max }
\end{array}\right], \forall p \in P e \quad \text { Eq. } 10
$$

Heat exchanger model and disjunctions. If the heat exchanger is "operating", the bypasses are disabled, and the heat transfer equations are enforced. Alternatively, if it is in a state of "being cleaned", the inlet flow rates are disabled, and the heat transfer equations are not applied. Eq. 11 is part of 'Continuous time representation', and Eq. 12 - Eq. 15 are part of 'HEX model'. 


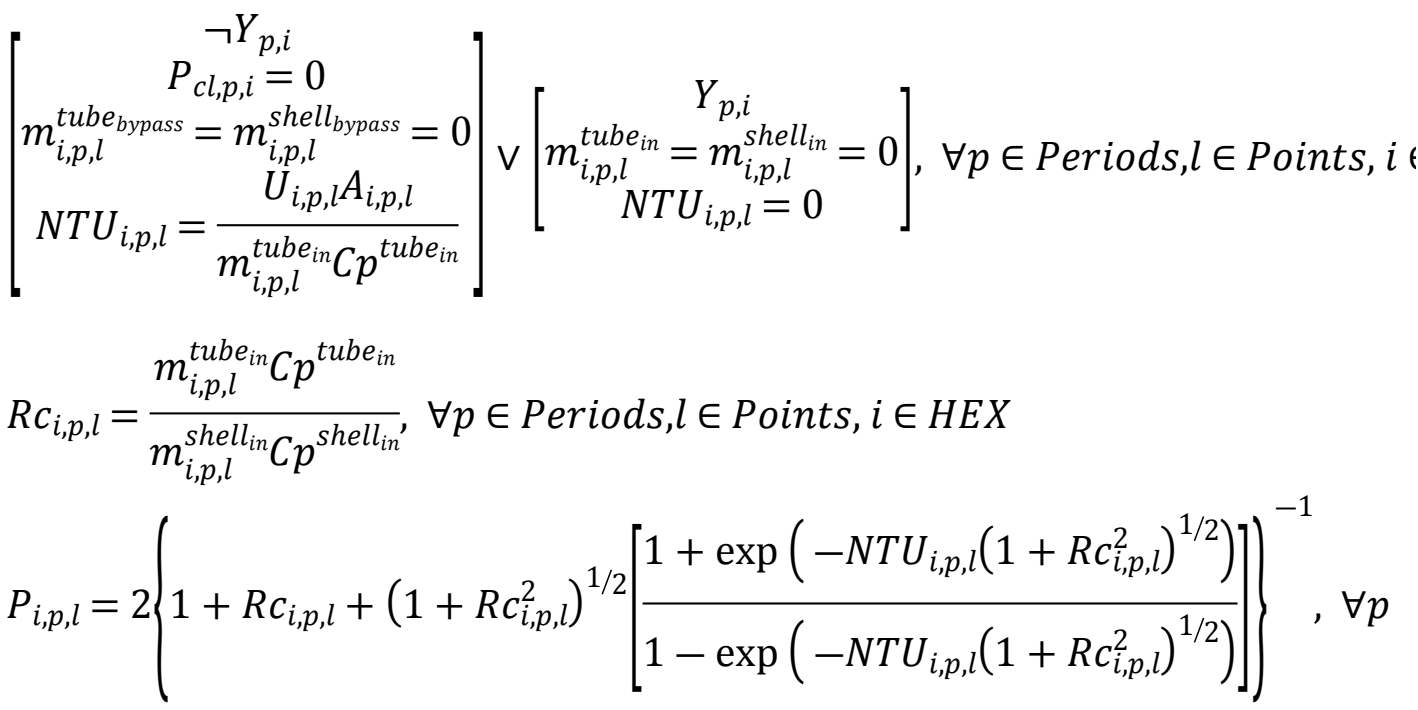

Eq. 11

Eq. 12

Eq. 13

$\in$ Periods, $l \in$ Points, $i \in$ HEX

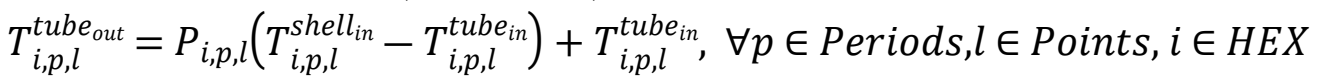

Eq. 14

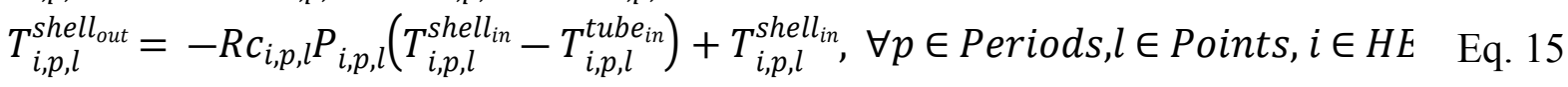

Fouling model, and disjunctions (included in Fouling model). When a heat exchanger state is "being cleaned" the fouling model does not apply, so that the fouling rate, and the fouling resistance are set to zero. When the state of the heat exchanger is "operating" the EbertPanchal fouling rate and the ageing models are applied.

$$
\begin{aligned}
& {\left[\frac{d R_{f, i, p, l}}{d \hat{t}}=\tau_{p}\left[\alpha_{i} R e^{-0.66} \mathrm{Pr}^{-0.33} \exp \left(-\frac{E_{f, i}}{R T_{f, i, p, l}}\right)-\gamma_{i} \tau_{w, i, p, l}\right]\right] \vee\left[\frac{d R_{f, i, p, l}}{d \hat{t}}=0\right], \forall p \quad \text { Eq. } 16} \\
& \in \text { Periods }, l \in \text { Points } \backslash\{0\}, i \in H E X \\
& {\left[\begin{array}{c}
\neg Y_{p, i} \\
R_{f, i, p, l}=R_{f, i, p-1, l^{\prime}}
\end{array}\right] \vee\left[\begin{array}{c}
Y_{p, i} \\
R_{f, i, p, l}=0
\end{array}\right], \forall p \in \operatorname{Periods} \backslash\{1\}, l \in\{0\}, l^{\prime} \in\left\{n_{t}\right\}, i \in H E X \quad \text { Eq. } 17} \\
& R_{f, i, p, l}=R_{f}^{0}, \forall p \in\{1\}, l \in\{0\}, i \in H E X
\end{aligned}
$$

In the previous differential equations $\hat{t}$ is a dimensionless time between zero and one used to standardize all the differential variables among the periods. Between two consecutive periods whether the differential variables may be continuous or not depends on the task assigned to those periods. 
Pressure drop model, and disjunctions (included in Pressure drop model). Only the pressure drop of the tube side is considered because it is assumed that fouling only occurs on the tube side. The pressure drop is a function of the available flow are, and the roughness of the deposit. It is defined as a disjunction based on the operating mode of the exchanger.

$\left[\Delta P_{i, p, l}=\frac{G_{t, i, p, l}^{2}}{2 \rho_{t, i, t, p, l}}\left(\frac{f_{p, i}}{2 R_{t}-2 \delta_{i, p, l}}\right) N_{P, i}\right] \vee\left[\begin{array}{c}Y_{p, i} \\ \Delta P_{i, p, l}=0\end{array}\right], \forall p \in$ Periods, $l \in$ Points $\}, i \in l$ Eq. 19 Complementarity constraints. This set of constrains refers to the reformulation of the MINLP as a mathematical program with complementarity constraints. First, the disjunctions are modelled as the bilinear product of continues variables or expression and a binary variable ( $W_{p, i, c} \in\{0,1\}, \forall p \in$ Periods, $i \in H E X, c \in$ Cleaning types $)$. Then, the binary variables are relaxed, slack variables introduced, and complementarity constraints imposed.

$0 \leq w_{i, p, c} \perp s_{i, p, c}^{b} \geq 0, \forall i \in H E X, p \in$ Periods,c $\in$ Cleaning types $\quad$ Eq. 20

$0 \leq\left(1-w_{i, p, c}\right) \perp s_{i, p, c}^{a} \geq 0, \forall i \in H E X, p \in$ Periods,c $\in$ Cleaning types $\quad$ Eq. 21

$0 \leq s_{i, p, c}^{a}, s_{i, p, c}^{b} \leq 1, \forall i \in H E X, p \in$ Periods,c $\in$ Cleaning types $\quad$ Eq. 22

\section{Additional information about the case study}

This section includes supplementary information about the definition and specification of the case study.

Table S1 lists the key specifications of the heat exchangers in the network. These are the main geometrical specifications of the shells required in the heat exchanger models. 
Table S1. Exchanger specifications for the HEN in Figure 4 of the manuscript. Adapted from [3].

\begin{tabular}{lrrrrrr}
\hline Shell & $\begin{array}{c}\text { Shell } \\
\text { diameter }[\mathbf{m m}]\end{array}$ & $\begin{array}{c}\text { No. } \\
\text { baffles [-] }\end{array}$ & $\begin{array}{c}\text { No. } \\
\text { tubes [-] }\end{array}$ & $\begin{array}{c}\text { No. passes } \\
{[-]}\end{array}$ & $\begin{array}{c}\text { Cleaning } \\
\text { time [days] }\end{array}$ & $\begin{array}{c}\text { Cleanings } \\
\text { cost [\$] }\end{array}$ \\
\hline E01A & 1245 & 5 & 764 & 2 & 9 & 27000 \\
\hline E01B & 1194 & 7 & 850 & 2 & 9 & 27000 \\
\hline E02A & 1397 & 18 & 850 & 4 & 10 & 30000 \\
\hline E02B & 1397 & 18 & 880 & 4 & 10 & 30000 \\
\hline E03A & 990 & 20 & 630 & 2 & 8 & 24000 \\
\hline E03B & 990 & 20 & 630 & 4 & 8 & 24000 \\
\hline E04 & 1270 & 9 & 888 & 4 & 9 & 27000 \\
\hline E05A & 1397 & 18 & 880 & 4 & 16 & 48000 \\
\hline E05B & 1397 & 18 & 880 & 4 & 16 & 48000 \\
\hline
\end{tabular}

Table S2 presents the solution of the parameter estimation problem using the data collected between 700 and 1200 days of operation, in order to validate the prediction capabilities of the model. These parameters are used to simulate the operation of the 'real' plant, but they are not visible to the prediction models, generate a model-plant mismatch. In that case, the parameters of the prediction models are estimated based on the current data every time an optimization problem is solved.

Table S2. HEN model parameters obtained from the validation with plant data (off line). Section 6.1 of the manuscript.

\begin{tabular}{lcccrr}
\hline Shell & $\begin{array}{c}\boldsymbol{\alpha} \\
{\left[\boldsymbol{m}^{\mathbf{2}} \boldsymbol{K} / \boldsymbol{W}\right]}\end{array}$ & $\begin{array}{c}\boldsymbol{\gamma} \\
{\left[\boldsymbol{m}^{\mathbf{4}} \boldsymbol{K} / \boldsymbol{W} \boldsymbol{N}\right]}\end{array}$ & $\begin{array}{c}\boldsymbol{E}_{\boldsymbol{f}} \\
{[\boldsymbol{J} / \boldsymbol{m o l} \boldsymbol{K}]}\end{array}$ & $\begin{array}{c}\boldsymbol{\varepsilon} \\
{[\boldsymbol{m m}]}\end{array}$ & $\begin{array}{l}\boldsymbol{R}_{\boldsymbol{f}}(\boldsymbol{t}=\mathbf{0}) \\
{\left[\boldsymbol{m}^{\mathbf{2}} / \boldsymbol{W}\right]}\end{array}$ \\
\hline E01A & $3.215 \times 10^{-3}$ & $4.838 \times 10^{-12}$ & 28500 & 0.084 & $1.37 \times 10^{-4}$ \\
\hline E01B & $6.230 \times 10^{-3}$ & $1.759 \times 10^{-12}$ & 28500 & 0.051 & $3.31 \times 10^{-3}$ \\
\hline E02A & $3.459 \times 10^{-3}$ & $6.840 \times 10^{-12}$ & 28500 & 0.053 & 0 \\
\hline E02B & $3.713 \times 10^{-3}$ & $7.662 \times 10^{-12}$ & 28500 & 0.042 & 0 \\
\hline E03A & $1.384 \times 10^{-3}$ & $2.882 \times 10^{-12}$ & 28500 & 0.030 & $1.22 \times 10^{-3}$ \\
\hline E03B & $1.198 \times 10^{-3}$ & $2.072 \times 10^{-12}$ & 28500 & 0.029 & $1.26 \times 10^{-3}$ \\
\hline E04 & $2.112 \times 10^{-3}$ & $1.817 \times 10^{-12}$ & 28380 & 0.041 & $3.31 \times 10^{-2}$ \\
\hline E05A & $3.552 \times 10^{-3}$ & $6.887 \times 10^{-12}$ & 28500 & 0.040 & 0 \\
\hline E05B & $3.881 \times 10^{-3}$ & $8.414 \times 10^{-12}$ & 28500 & 0.032 & 0 \\
\hline
\end{tabular}




\section{References}

[1] F. Lozano Santamaria and S. Macchietto, "Integration of Optimal Cleaning Scheduling and Control of Heat Exchanger Networks Undergoing Fouling: Model and Formulation," Ind. Eng. Chem. Res., vol. 57, no. 38, pp. 12842-12860, Sep. 2018.

[2] F. L. Santamaria and S. Macchietto, "Integration of optimal cleaning scheduling and control of heat exchanger networks under fouling: MPCC solution," Comput. Chem. Eng., vol. 126, pp. 128-146, Jul. 2019.

[3] F. Coletti and S. Macchietto, "Refinery Pre-Heat Train Network Simulation Undergoing Fouling: Assessment of Energy Efficiency and Carbon Emissions,” Heat Transf. Eng., vol. 32, no. 3-4, pp. 228-236, Mar. 2011. 